\title{
Piezo2: A Candidate Biomarker for Visceral Hypersensitivity in Irritable Bowel Syndrome?
}

\author{
Tao Bai, Ying Li, Jing Xia, Yudong Jiang, Lei Zhang, Huan Wang, Wei Qian, Jun Song, ${ }^{*}$ and Xiaohua Hou \\ Division of Gastroenterology, Union Hospital, Tongji Medical College, Huazhong University of Science and Technology, Wuhan, China
}

\begin{abstract}
Background/Aims
Currently, there exists no biomarker for visceral hypersensitivity in irritable bowel syndrome (IBS). Piezo proteins have been proven to play an important role in the mechanical stimulation to induce visceral pain in other tissues and may also be a biomarker candidate. The aim of this study was to test the expressions of Piezo1 and Piezo2 proteins in the intestinal epithelial cells from different intestinal segments and to explore the correlation between Piezo proteins expression and visceral pain threshold.
\end{abstract}

\section{Methods}

Post-infectious IBS was induced in mice via a Trichinella spiralis infection. Visceral sensitivity was measured with abdominal withdrawal reflex to colorectal distention. Inflammation in the small intestine and colon was scored with H\&E staining. Expression location of Piezo proteins was confirmed by immunohistochemistry. Abundance of Piezo proteins were measured with real-time reverse transcriptase polymerase chain reaction.

\section{Results}

Piezo1 and Piezo2 proteins were expressed in the intestinal epithelial cells. The expression levels of Piezo1 and Piezo2 were abundant in the colon than the small intestine $(P<0.001$ for Piezo1, $P=0.003$ for Piezo2). Expression of Piezo2 in the colon significantly correlated to the visceral sensitivity $(r=-0.718, P=0.001)$ rather than the mucosal inflammation.

\section{Conclusion}

Piezo2 is a candidate biomarker for visceral hypersensitivity in IBS.

(J Neurogastroenterol Motil 2017;23:453-463)

\section{Key Words}

Hyperalgesia; Ion channels; Irritable bowel syndrome; Pizeo1 protein, human; Piezo2 protein, human

\section{Introduction}

Irritable bowel syndrome (IBS) is a common functional gastrointestinal disorder worldwide. The prevalence of IBS varies from $10 \%$ to $20 \%$ in different countries. ${ }^{1-3}$ Although IBS poses no risk to life, chronic and recurrent symptoms of IBS greatly influence patients' quality of life. ${ }^{4}$ Visceral hypersensitivity is one of the most important mechanisms of IBS, which is generally considered as a hallmark of IBS. ${ }^{5}$ With lower thresholds for pain as well as

Received: July 18, 2016 Revised: October 17, 2016 Accepted: October 23, 2016

() This is an Open Access article distributed under the terms of the Creative Commons Attribution Non-Commercial License (http://creativecommons. org/licenses/by-nc/4.0) which permits unrestricted non-commercial use, distribution, and reproduction in any medium, provided the original work is properly cited.

${ }^{*}$ Correspondence: Jun Song, MD

Division of Gastroenterology, Union Hospital, Tongji Medical College, Huazhong University of Science and Technology, 1277 Jiefang Road, Wuhan 430022, China

Tel: +86-27-85726057, Fax: +86-27-85726057,E-mail: song111jun@126.com 
increased intensity of sensations, visceral hypersensitivity makes a great contribution to abdominal pain, which is the most predominant and distressing symptom of IBS. ${ }^{6}$

Diagnosis of visceral hypersensitivity is generally performed with barostat controlled rectal distention. ${ }^{7}$ The feeling and pain thresholds were evaluated. However, it was an independent invasive examination among all the other screening tests for the organic diseases. Therefore, it is meaningful to combine evaluation of visceral hypersensitivity with other screening tests to reduce patient's suffering and expenditure. Since 12.7-32.1\% patients with symptoms compatible with IBS exhibited organic gastrointestinal diseases, ${ }^{8}$ colonoscopy is still a valuable test for IBS, especially for patients with alarming symptoms or initial diagnosis. We expected to find a biomarker of visceral hypersensitivity locally expressed in intestinal epithelial cells, which could be tested with biopsies obtained under colonoscopy.

Recent studies have proven that Piezo proteins are important mechanical gated ion channels. ${ }^{9}$ Piezo proteins comprising Piezo1 and Piezo2, are expressed in the cell membrane and the Piezo channels opened following mechanical stimulation. ${ }^{9}$ Unselected positive ions influx causes the change of the membrane potential, and then induces the changes of downstream signaling pathways responding to mechanical stimulation. ${ }^{10}$ Piezo proteins are expressed in different species and tissues conservatively. ${ }^{9}$ Previous studies have shown that Piezo proteins were also expressed in the endothelium of blood vessels and play an important role in endothelial pain. ${ }^{11}$ On the basis that intestinal epithelium cells also live in a complex mechanical microenvironment ${ }^{12}$ as blood vessel endothelium, the question arises - are there any possibilities that Piezo proteins take part in the mechanical stimulation to induce visceral pain in intestinal tract and therefore act as a candidate biomarker of visceral hypersensitivity for IBS?

The aims of our study are: (1) to test the expressions of Piezo1 and Piezo2 in intestinal epithelial cells of different intestinal segments, (2) to compare the expressions of Piezo1 and Piezo2 in mice with and without visceral hypersensitivity, and (3) to explore the correlation between Piezo proteins expression and visceral pain threshold.

\section{Materials and Methods}

\section{Animals}

Male NIH mice (6-8 weeks old) were ordered from the Medical Animal Laboratory Center of Guangdong. Mice were raised under specific pathogen-free conditions at the Animal Laboratory Center of Tongji Medical College, Huazhong University of Science and Technology. All experimental procedures were approved by Ethics Committee of the Tongji Medical College.

\section{Model of Visceral Hypersensitivity}

We performed the animal model of visceral hypersensitivity as mentioned previously. ${ }^{13}$ Trichinella spiralis were obtained from the Department of Parasitology, Huazhong University of Science and Technology. The colony was maintained in infected SpragueDawley rats. We performed a modified technique to obtain the larvae from infected rodents as described by Castro. ${ }^{14} T$. spiralis larvae were counted under a microscope. Each mouse was infected by gavaging of 350 larvae in a $0.2 \mathrm{~mL}$ of phosphate-buffered saline.

\section{Study Design}

Twenty-two mice were randomly divided into 3 groups: control $(\mathrm{n}=6)$, acute infection group ( 2 weeks post-infection; $\mathrm{n}=8)$, and post-infection group ( 8 weeks post-infection; $\mathrm{n}=8$ ). To evaluate the model of visceral hypersensitivity, body weight changes, histopathology inflammation scores, and visceral sensitivity assessing were tested. Immunohistochemistry was performed to assure the expression of Piezo proteins in the intestinal epithelial cells. Realtime polymerase chain reaction (PCR) was performed to compare the mRNA transcription of PIEZO1 and PIEZO2 in intestinal epithelial cells of different intestinal segments and to further compare the expressions of Piezo1 and Piezo2 in mice with and without visceral hypersensitivity.

\section{Abdominal Withdrawal Reflex Recording to Colorectal Distention}

Colorectal distention was performed as described previously. ${ }^{15}$ Abdominal withdrawal reflex was recorded during plastic balloon inflation to $20,40,60$, and $80 \mathrm{mmHg}$. Score scale of the abdominal withdrawal reflex has been described previously. Threshold intensity of colorectal distention was recorded when the stimulus intensity evoked a visually identifiable contraction of the abdominal wall. Colorectal distention was performed in mice for 20 seconds every 4 minutes. Two investigators observed the abdominal withdrawal reflex independently and balloon inflation was done 5 times to achieve an accurate result.

\section{Histopathological Study}

Intestinal segments were removed and placed in $4 \%$ paraformaldehyde, embedded in paraffin and cut. $\mathrm{H} \& \mathrm{E}$ staining was per- 
formed by standard techniques. Inflammation scores of the intestine were graded as described previously ${ }^{16}: 1+$ mild, infiltration of a limited number of neutrophils in the lamina propria with minimal or no interstitial edema; $2+$ moderate, infiltration of a moderate numbers of neutrophils in the lamina propria and moderate interstitial edema; $3+$ severe, diffuse infiltration of moderate to large numbers of neutrophils in the lamina propria with severe interstitial edema. Two investigators independently assessed the inflammation scores and the mean of total scores were obtained.

\section{Immunohistochemistry}

Intestinal segments were removed and placed in $4 \%$ paraformaldehyde, embedded in paraffin and cut. Immunohistochemistry was performed by standard techniques. The specimens were blocked with $5 \%$ bovine serum albumin and then incubated with rabbit anti-Piezo1 (1:250; Abcam, Cambridge, MA, USA) or rabbit anti-Piezo2 (1:250; Abcam) as the primary antibody overnight at $4^{\circ} \mathrm{C}$. After incubation with the appropriate biotinylated secondary antibody for 30 minutes at room temperature, the results were visualized using diaminobenzidine (DAB). The densitometry analysis of immunohistochemical staining was performed using the Image-Pro Plus 6.0 software (Version X; Media Cybernetics, Silver Springs, MD, USA).

\section{Real-time Reverse Transcription Polymerase Chain Reaction}

Total cellular RNA were extracted from the intestinal segments with Trizol (Gibco, Grand Island, NY, USA) using a standard method according to the manufacturer. An aliquot ( $\mu \mathrm{g})$ of RNA was reverse-transcribed into first-strand complementary DNA (cDNA) as the manufacture's recommendation of Takara Kits (Takara Biomedicals, Tokyo, Japan). Real-time reverse transcriptase polymerase chain reaction (RT-PCR) was carried out with a light cycler using DNA-binding dye SYBERGreen for detection of

Table. Primer Pairs

\begin{tabular}{cll}
\hline Gene & & \multicolumn{1}{c}{ Sequence (5'-3') } \\
\hline Piezo1 & Forward & TCATCATCCTTAACCACATGGTG \\
& Reverse & TGAAGACGATAGCTGTCATCCA \\
Piezo2 & Forward & GTGGTATGCAACCCAGTACCC \\
& Reverse & GGCCATTCTCTATGGGCAGG \\
\multirow{3}{*}{-actin } & Forward & TGTTACCAACTGGGACGACA \\
& Reverse & CTGGGTCATCTTTCACGGT
\end{tabular}

PCR products. The reaction mixture contained $5 \mu \mathrm{L}$ of SYBERGreen, $3 \mu \mathrm{L}$ RNAase free water, $1 \mu \mathrm{L}$ primer, and $1 \mu \mathrm{L}$ cDNA to give a final reaction volume of $10 \mu \mathrm{L}$. The primer pairs are shown in the tabulated form below (Table).

\section{Statistical Methods}

Abdominal withdrawal reflex scores at each pressure of colorectal distention as well as the inflammation scores between control and model groups were expressed as median (interquartile range) and compared with Kruskal-Wallis test. A Wilcoxon rank sum test was performed with a Bonferroni correction at $0.05 / 3$ to correct for multiple comparisons. Other data were expressed as means \pm standard errors and compared with $t$ test or variance analysis. Pearson's correlation was performed to assess the relationship between expression of Piezos and visceral sensitivity. Statistical analyses were performed using R 2.11.1 software (R Foundation for Statistical Computing, Vienna, Austria). The information is available from URL: https:/www.r-project.org/doc/R-SDLC.pdf (accessed 7 June, 2017). GraphPad Prism 5 software (GraphPad, Version X; La Jolla, CA, USA) was used for all graph creation. A $P$-value of $<0.05$ was considered significant.

\section{Results}

\section{Animal Model}

Three mice died from $T$. spiralis infection at the first week and one mouse died from abdominal withdrawal reflex recording. Eighteen mice were included in the following experiments (control group, $\mathrm{n}=6$; acute infection group, $\mathrm{n}=6$; post-infection group, $\mathrm{n}=6$ ). Weight gain of the mice in the infection group was slower than that in the control group. Weights of mice in the infection group and the control group were significantly different $(P=0.011)$ (Fig. 1A). As for visceral sensation, mice presented with increased visceral sensation and decreased pain threshold after infection compared with the control group (Fig. 1B and 1C). During the acute infection period ( 2 weeks post-infection), significant increase of abdominal withdrawal reflex scores was observed compared with the control mice for intensities of 20, 40, and $60 \mathrm{mmHg}(P<0.001)$. Lower pain threshold was shown in mice with acute infection compared with control mice $(P<0.001)$. However, compared with the control mice, the abdominal withdrawal reflex scores remained at high level relatively $(P<0.001)$ and the pain threshold was still low $(P<0.001)$ in mice of the post-infection group ( 8 weeks post- 
A

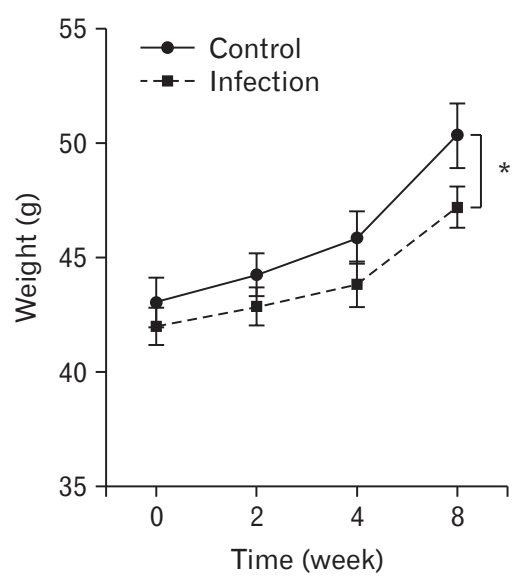

B

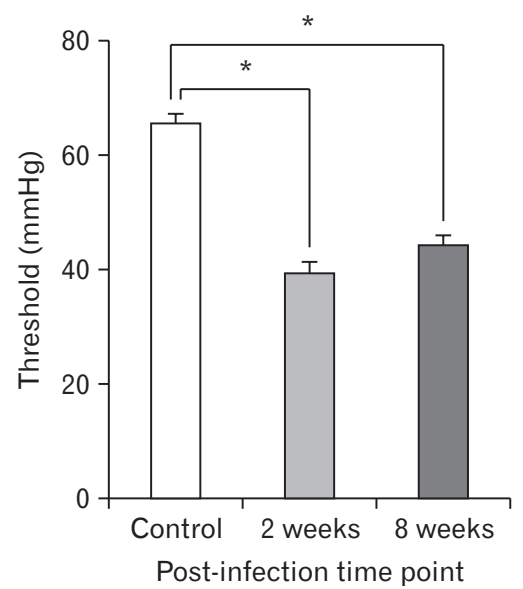

C
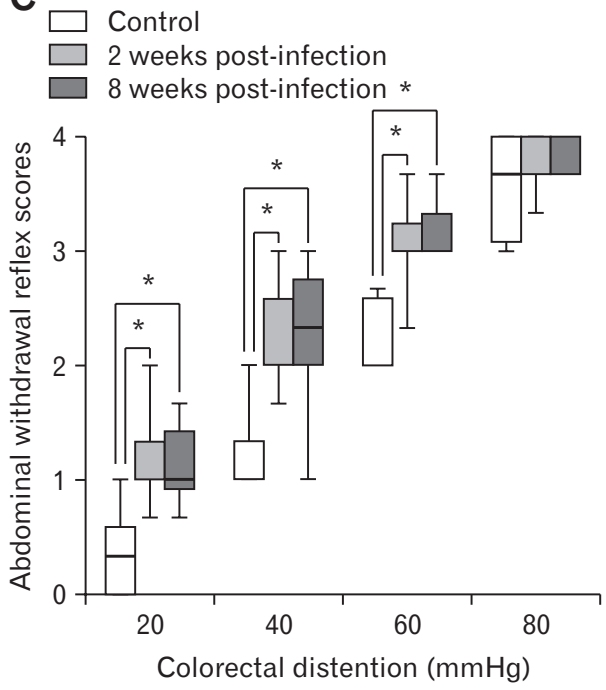

Figure 1. Weight, abdominal withdrawal reflex scores, and thresholds of control and post-infectious irritable bowel syndrome (PI-IBS) mouse model. (A) Weight of control and PI-IBS mice $\left({ }^{*} P=0.011\right)$. (B) Thresholds of the colorectal distention intensities that evoke abdominal contraction of the mice. Mean \pm SEM values were plotted $\left({ }^{\star} P<0.001\right)$. (C) Box plot of abdominal withdrawal reflex scores. Lines represent the median within the box, the 25 th and 75 th centiles at the ends of the box, and the error bars define the 5 th and 95 th centiles $\left({ }^{\star} P<0.001\right)$.
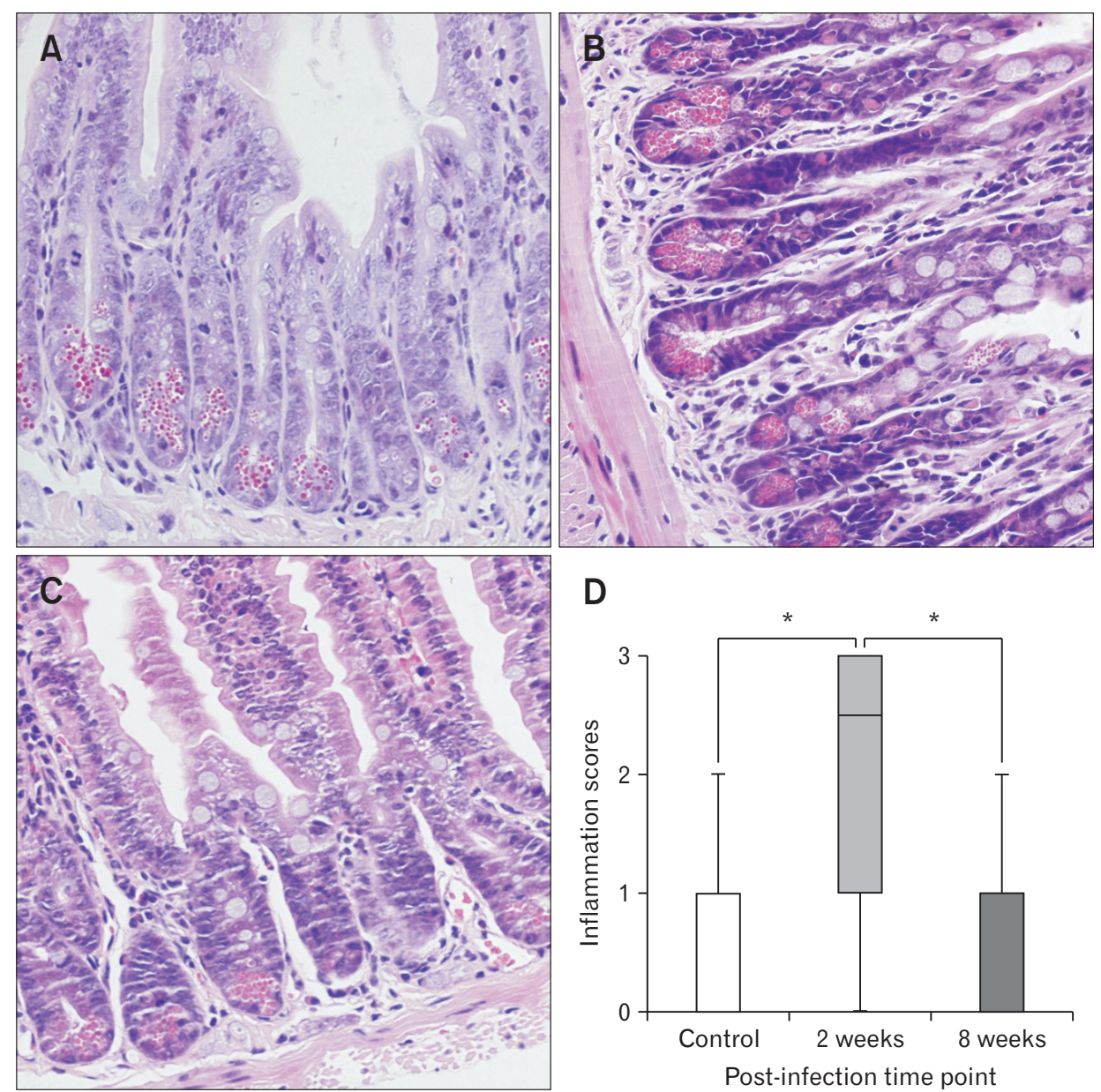

D

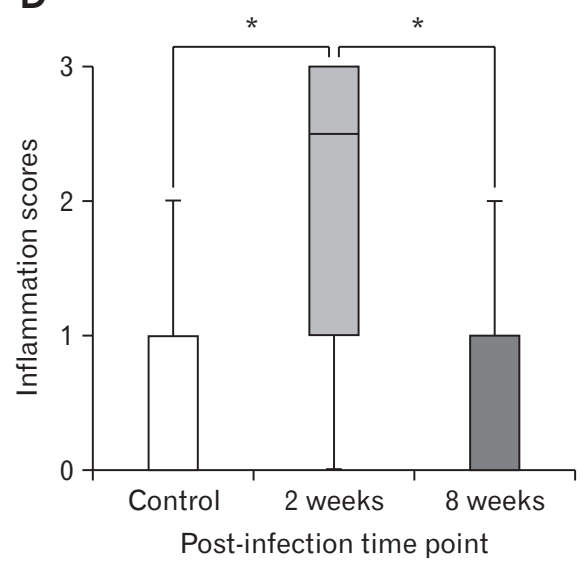

Figure 2. Inflammation scores in $\mathrm{H} \& \mathrm{E}$ stained sections of small intestine. (A) H\&E stained sections of small intestine from control mice. (B) H\&E stained sections of small intestine from mice with acute infection. (C) H\&E stained sections of small intestine from mice with post-infection. (D) Inflammation scores. Lines represent the median within the box, the 25 th and 75 th centiles at the ends of the box, and the error bars define the 5 th and 95 th centiles. ${ }^{*} P<0.001$. 

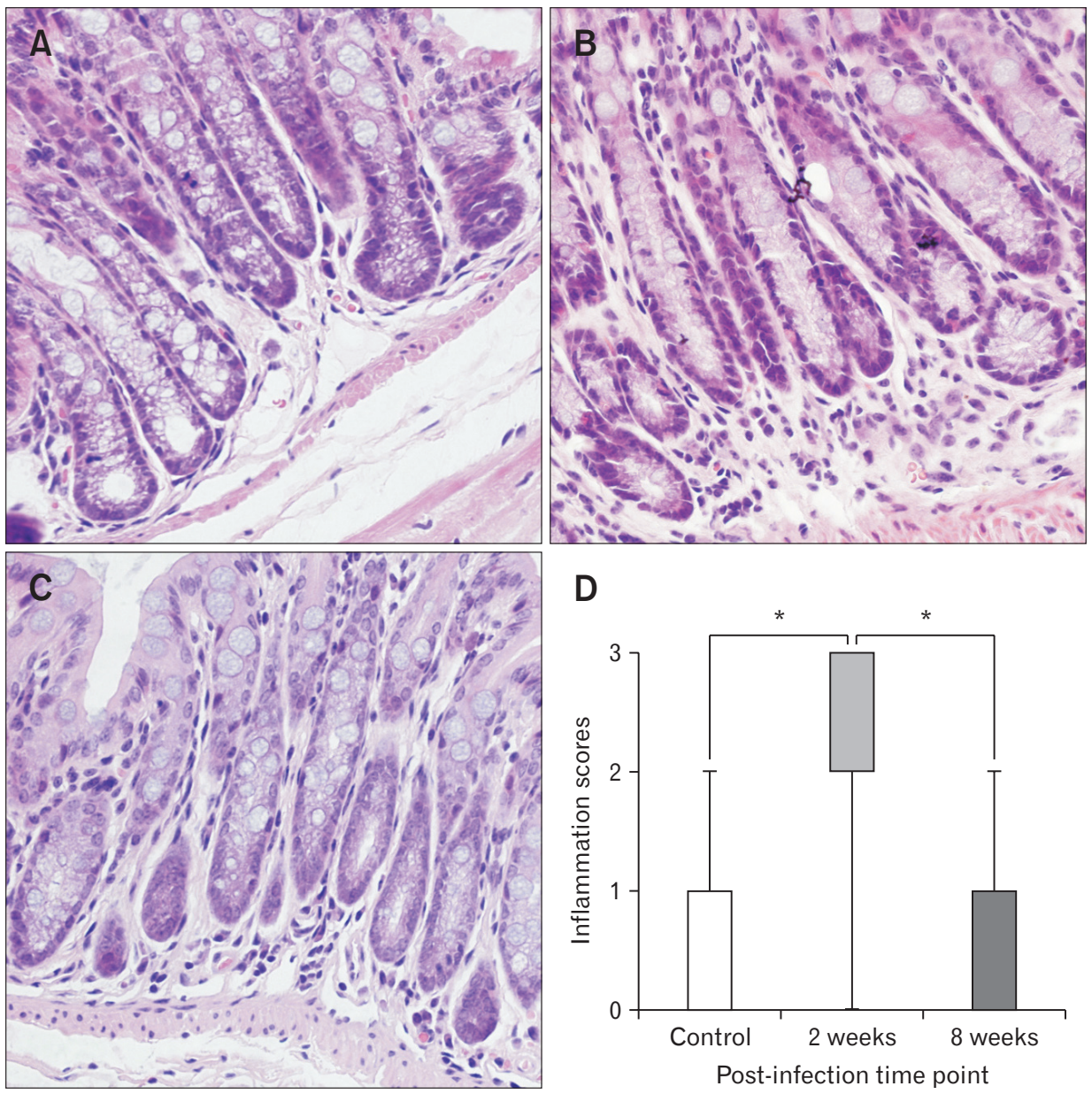

Figure 3. Inflammation scores in $\mathrm{H} \& \mathrm{E}$ stained sections of colon. (A) $\mathrm{H} \& \mathrm{E}$ stained sections of colon from control mice. (B) H\&E stained sections of colon from mice with acute infection. (C) H\&E stained sections of colon from mice with post-infection. (D) Inflammation scores. Lines represent the median within the box, the 25th and 75 th centiles at the ends of the box, and the error bars define the 5 th and 95 th centiles. ${ }^{*} P<0.001$.

infection). No significant difference of abdominal withdrawal reflex scores or pain threshold was shown between mice in the acute infection group and the post-infection group. This suggests that all mice with $T$. spiralis infection had visceral hypersensitivity in both the acute infection phase as well as the post-infection phase.

\section{Mucosal Histopathology}

At the acute infection phase, obvious lesions and inflammation presented in the small intestine (Fig. 2) and colon (Fig. 3), with severe hyperemia and swelling on macroscopic observation and also severe eosinophilic and neutrophilic infiltration in the lamina propria. Higher inflammation scores were evaluated in both the small intestine $(P<0.001)$ and colon $(P<0.001)$ of mice with acute infection $(n=6)$ compared with the control groups $(n=6)$. However, the inflammation almost disappeared at the microscopic level in both the small intestine as well as in the colon of mice in the postinfection group $(n=6)$. Inflammation scores of the small intestine
(Fig. 2) and colon (Fig. 3) in mice of the post-infection group ( $\mathrm{n}=$ 6 ) did not differ from controls $(n=6)$.

\section{Expressions of Piezo Proteins in Intestine}

As shown in Figures 4 and 5, both Piezol and Piezo2 are expressed in intestinal epithelial cells. Immunohistochemistry staining and real-time RT-PCR showed that expressions of Piezol and Piezo2 were much more abundant in colon rather than small intestine (Fig. 4E, 5E, 6A, and 6B). We observed the expression of proteins (Fig. 4F and 5F) and mRNA in the small intestine and colon of mice in different groups (Fig. 6C-F). In the small intestine, although Piezo1 and Piezo2 were highly expressed in mice with infection, there were no significant differences among the other 3 groups ( $n=6$ in each group). In the colon, Piezol mRNA was expressed more abundantly in mice of the post-infection group rather than that in the acute infection group $(P=0.040)$ or the control group $(P$ $=0.017)$. Results of immunohistochemistry staining showed po- 

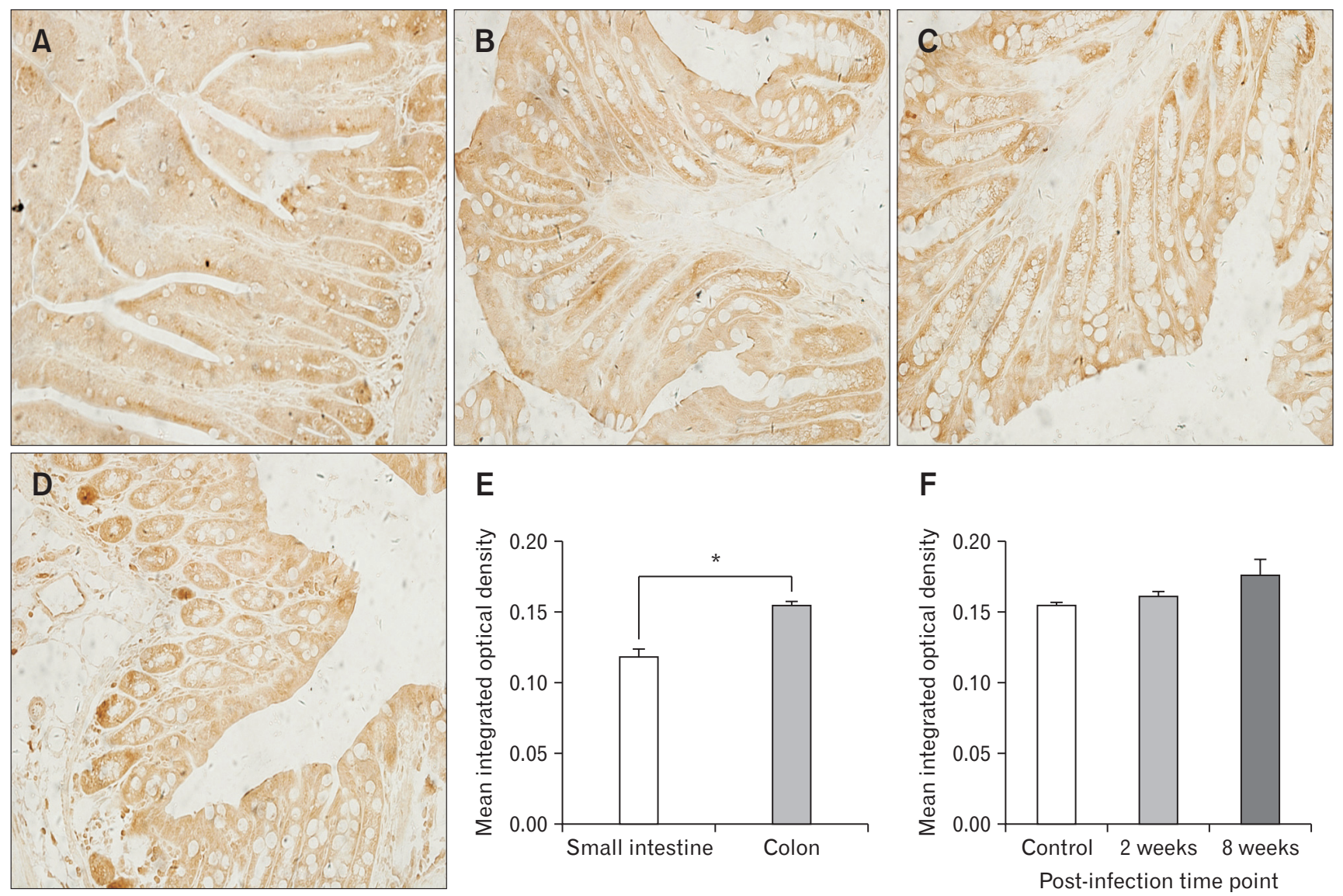

Figure 4. Immunohistochemistry staining for Piezo1. (A) Expression of Piezo1 in control group small intestine. (B) Expression of Piezo1 in the colon of mice in control group. (C) Expression of Piezo1 in the colon of mice in acute infection group. (D) Expression of Piezo1 in the colon of mice in post-infection group. (E) Comparison of Piezo1 expression in control group small intestine and colon. (F) Comparison of Piezo1 expression in control, acute infection, and post-infection groups. ${ }^{\star} P<0.05$.

tential differences without significance. On the other hand, Piezo2 was expressed abundantly in mice of both acute infection group ( $P$ $=0.034$ and 0.019 for $\mathrm{mRNA}$ and protein, respectively) and postinfection group $(P=0.024$ and 0.047 for $\mathrm{mRNA}$ and protein, respectively) rather than the controls group ( $\mathrm{n}=6$ in each group) on the level of both mRNA and protein.

\section{Correlation between Piezos and Visceral Sensation}

To explore whether the visceral sensation could be marked with expressions of Piezo1 and Piezo2, the correlation between mRNA expressions of Piezo1/Piezo2 and pain threshold were analyzed (Fig. 7). No significant correlation was found between visceral sensitivity with Piezo1 or Piezo2 expression in the small intestine. Although potential negative correlation was established between Piezo1 expressed in the colon and visceral sensitivity, the $P$ - value was not significant. However, Piezo2 expressed in the colon showed a close and negative correlation with visceral sensitivity $(r$ $=-0.718, P=0.001)$.

\section{Discussion}

In this study we observed that the Piezo1 and Piezo2, the newly discovered mechanical gated ion channels were expressed in the intestinal epithelial cells in mice. The expression was more abundant in the colon than in the small intestine. A good correlation was observed between visceral sensitivity and expression of Piezo2 in the colon. It is suggested that Piezo2 might be a candidate biomarker for visceral hypersensitivity. To our knowledge, this is the first study on the relationship between expressions of Piezo proteins and visceral sensation in the gastrointestinal tract. Although the mechanism 

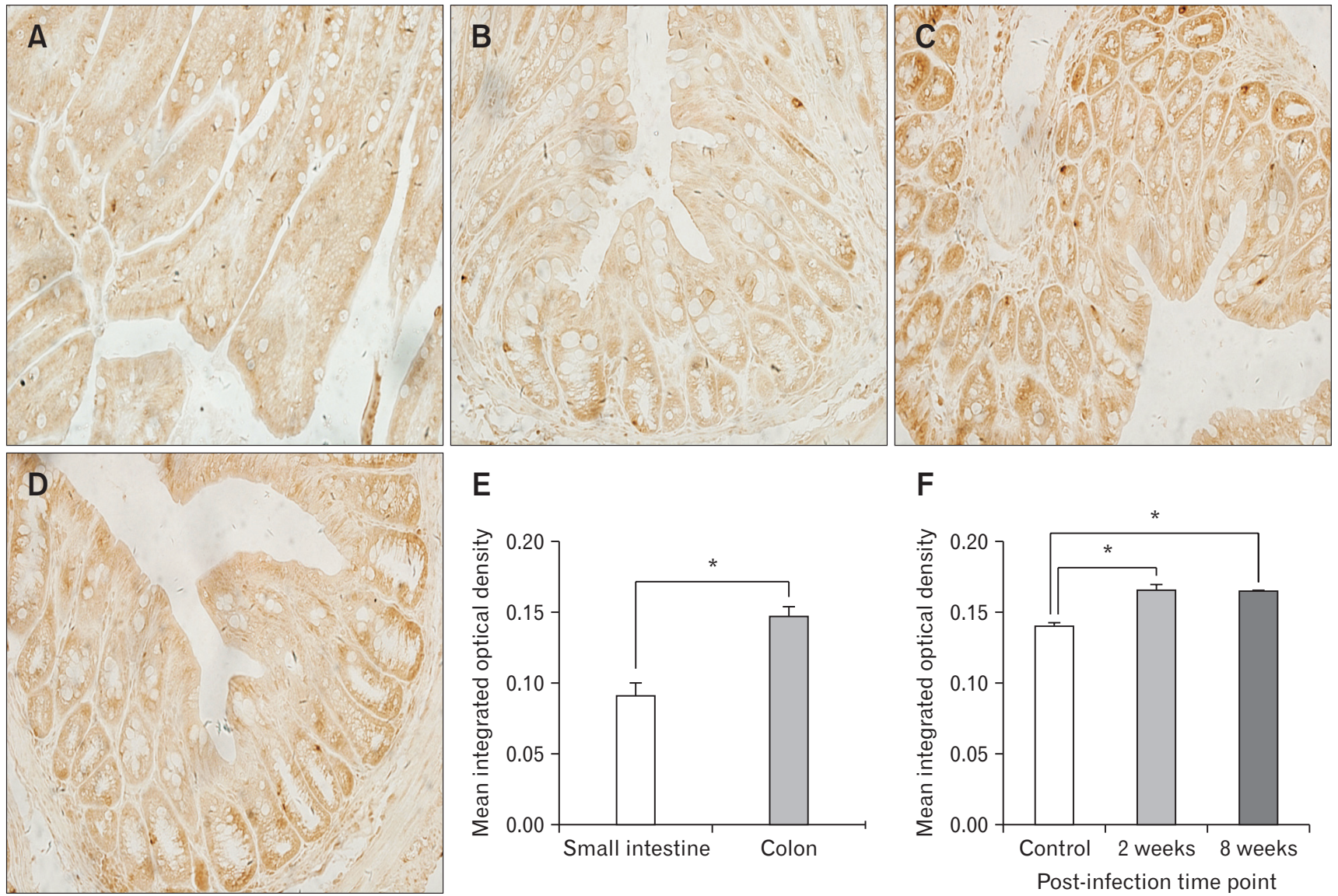

Figure 5. Immunohistochemistry staining for Piezo2. (A) Expression of Piezo2 in control group small intestine. (B) Expression of Piezo2 in the colon of mice in control group. (C) Expression of Piezo2 in the colon of mice in acute infection group. (D) Expression of Piezo2 in the colon of mice in post-infection group. (E) Comparison of Piezo2 expression in control group small intestine and colon. (F) Comparison of Piezo2 expression in control, acute infection, and post-infection groups. ${ }^{*} P<0.05$.

is unknown, the outcome gave us a new perspective to test visceral sensitivity.

As reported in previous studies, Piezo1 and/or Piezo2 are expressed among different species conservatively. ${ }^{9}$ Considering the electrophysiological characteristics of these ion channels, it was suggested that Piezo1 and Piezo2 might induce the basic mechanical stimulation in development and maturation. ${ }^{9}$ Our study initially proved that Piezo1 and Piezo2 were expressed in the intestinal epithelium cells. Intestinal epithelium cells live in a microenvironment with complex mechanical stimulation. ${ }^{12}$ Therefore, Piezo1 and Piezo2 might play an important role in sensation of mechanical stimulation as well as in the induction for maintaining the normal physiological status of the intestine. Limited studies have been conducted on the relationship between expression of ion channel molecules in the epithelium and sensation. However, increasing evi- dence has proven that the function of the intestinal epithelium was strongly related to visceral sensation. ${ }^{15,17,18}$ A recent study has shown that Piezo2 expressed in blood vessel endothelium might induce the sensation of endothelial pain. ${ }^{11}$ It was possible that Piezol and Piezo2 played a similar role of sensation in intestinal epithelial cells.

Our study showed that Piezo1 and Piezo2 were expressed more abundantly in the colon than in the small intestine. This was basically consistent with the complexity of microenvironment. In the colon, stool with various characteristics were formed and the microbial flora was more abundant and complicated than in the small intestine. ${ }^{19-22}$ Increasing expression of Piezo1 and Piezo2 might promote the sense to mechanical stimulation and induce the adaptability to changes of the mechanical microenvironment. We did not find any significant correlation between expression of Piezo1 or Piezo2 in the small intestine and visceral sensitivity. This 

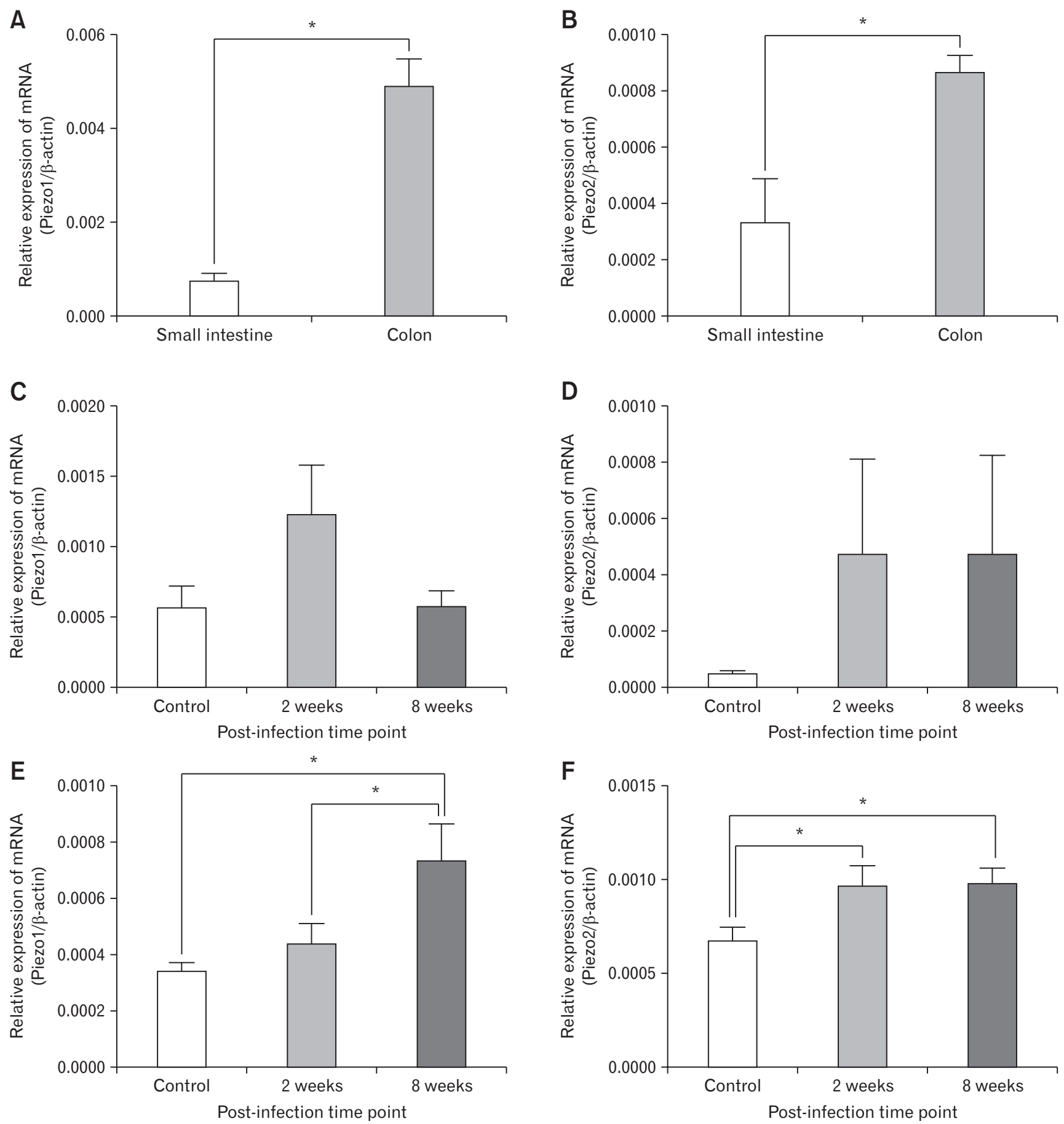

Fiǵure 6. Relative expression of Piezo1 and Piezo2. (A) Relative expression of Piezo1 in control group small intestine and colon. (B) Relative expression of Piezo2 in control group small intestine and colon. (C) Relative expression of Piezo1 in small intestine of mice in control, acute infection, and post-infection groups. (D) Relative expression of Piezo2 in small intestine of mice in control, acute infection, and post-infection groups. (E) Relative expression of Piezo1 in colon of mice in control, acute infection, and post-infection groups. (F) Relative expression of Piezo2 in colon of mice in control, acute infection, and post-infection groups. ${ }^{*} P<0.05$. 
A
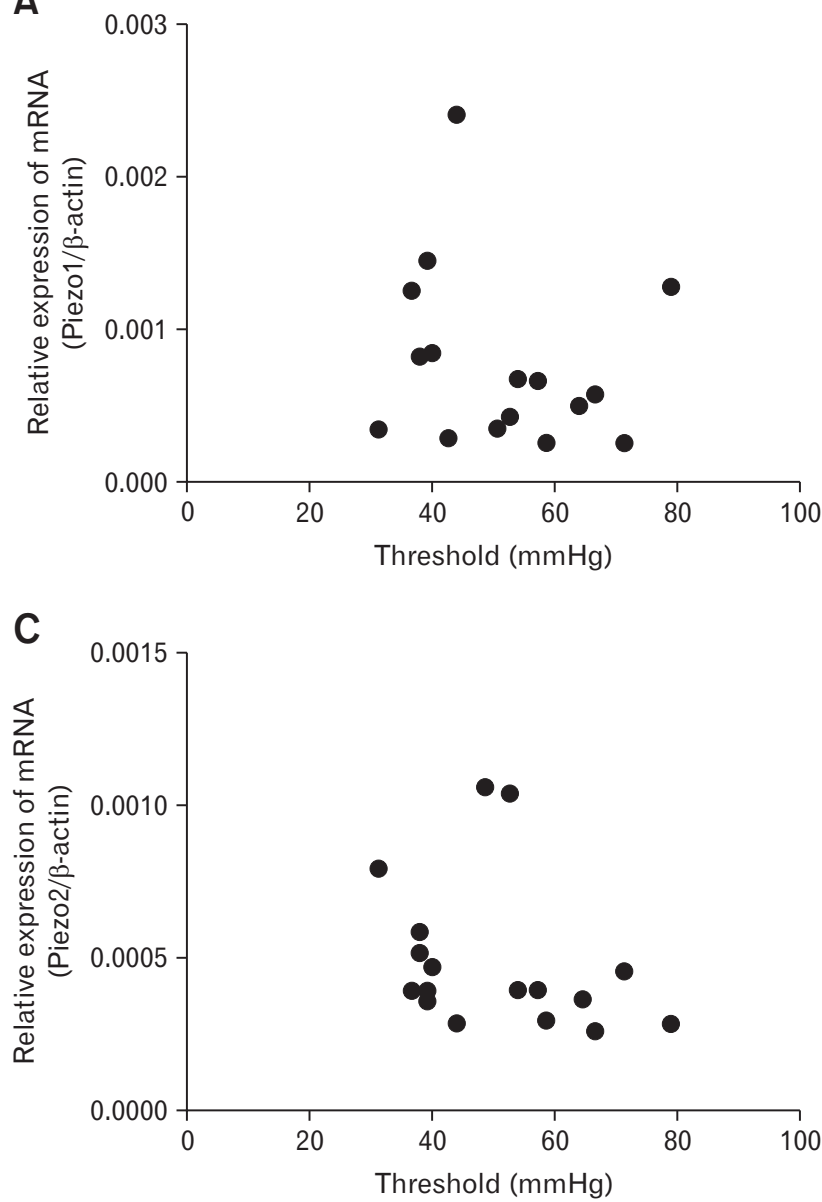

B
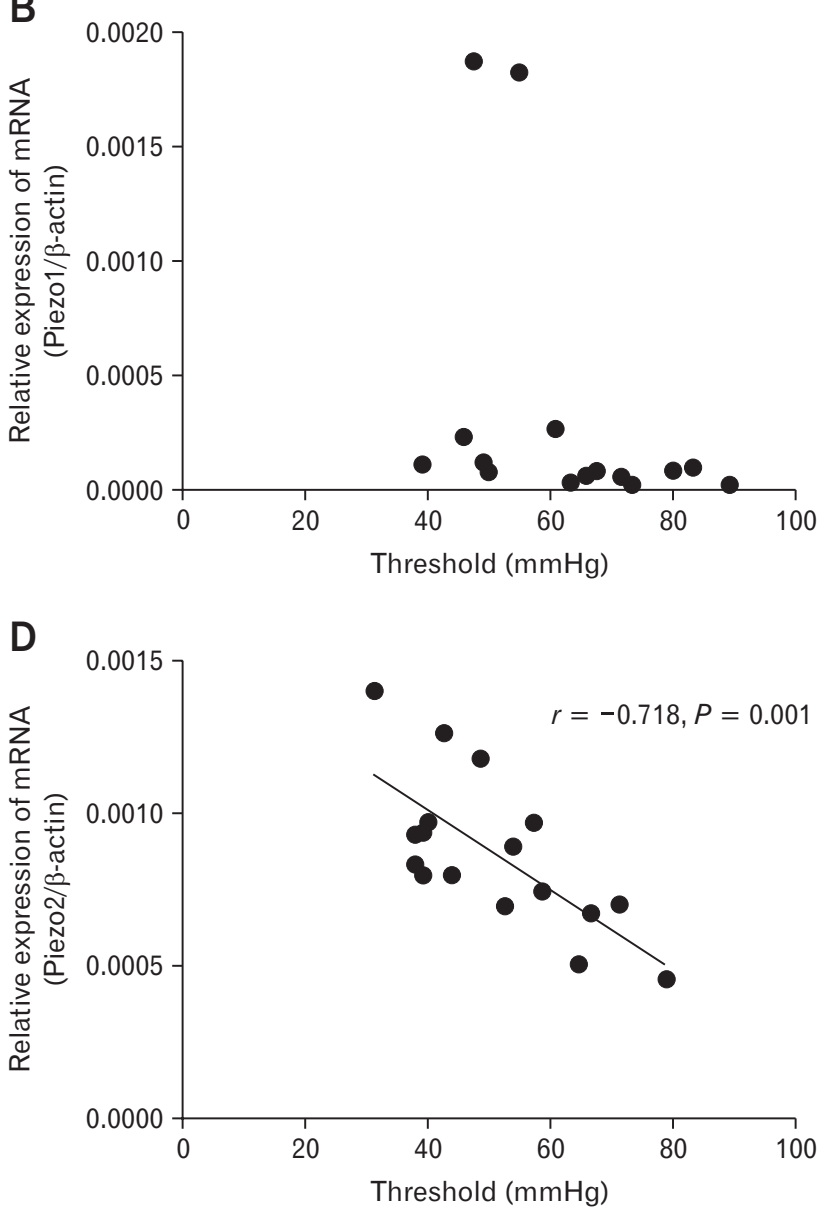

Figure 7. Correlation between visceral sensation and expression of Piezo1 and Piezo2. (A) Correlation between visceral sensation and expression of Piezol in small intestine samples from mice in control group, acute infection group, and post-infection group. (B) Correlation between visceral sensation and expression of Piezol in the colon samples from mice in control group, acute infection group, and post-infection group. (C) Correlation between visceral sensation and expression of Piezo2 in the small intestine samples from mice in control group, acute infection group, and postinfection group. (D) Correlation between visceral sensation and expression of Piezo2 in the colon samples from mice in control group, acute infection group, and post-infection group.

may be because of the limited abundance of the Piezo protein. On the other hand, visceral sensitivity was recorded by the abdominal withdrawal reflex to colorectal distention. No appropriate method was available to test the visceral sensitivity of the small intestine directly. Therefore, their correlation was poor in the small intestine. In the colon, significant correlation was found between expression of Piezo2 and visceral sensitivity. Although not significant, expression of Piezol had a potentially negative correlation to visceral sensitivity. In our model of visceral hypersensitivity, mice in the acute infection and post-infection groups had similar visceral sensitivities with almost the same thresholds. However, mice with acute infections had noticeable mucosal inflammation while the mucosa almost returned to normal in mice of the post-infection group. Therefore, the significant correlation between $\mathrm{Piezo} 2$ and visceral sensitivity was independent of mucosal inflammation. Recent studies have shown that Piezo2 was highly expressed selectively in enterochromaffin cells of the small intestine, which could sense mechanical stimulation and induce the release of 5-hydroxytryptamine (5-HT). ${ }^{23,24}$ Numbers of studies showed that 5-HT played a key role in visceral sensitivity in IBS. ${ }^{5,25,26}$ Therefore, changes of Piezo2 expression could influence visceral sensitivity via modulation of 5-HT release. This result greatly indicated that Piezo2 was a candidate biomarker for visceral hypersensitivity in colon.

In conclusion, Piezo1 and Piezo2 were expressed in intestinal 
epithelial cells. Expressions of Piezo1 and Piezo2 were expressed abundantly in the colon than in the small intestine. The expression of Piezo2 in the colon significantly correlated to visceral sensitivity which indicated Piezo2 may act as a candidate biomarker for visceral hypersensitivity in IBS.

Acknowledgements: We would like to thank our friend, Dr Stuti Sharma for helping us in editing this paper.

Financial support: This study was supported by a grant from The National Natural Science Foundation of China (NSFC) (Grant No. 81070300 and 81200271).

\section{Conflicts of interest: None.}

Author contributions: Xiaohua Hou and Jun Song designed the research study; Tao Bai, Wei Qian, Yudong Jiang, and Lei Zhang performed the experiment; Jing Xia and Huan Wang analyzed the data; and Tao Bai drafted the manuscript.

\section{References}

1. Wilson S, Roberts L, Roalfe A, Bridge P, Singh S. Prevalence of irritable bowel syndrome: a community survey. Br J Gen Pract 2004;54:495-502.

2. Gwee KA, Wee S, Wong ML, Png DJ. The prevalence, symptom characteristics, and impact of irritable bowel syndrome in an asian urban community. Am J Gastroenterol 2004;99:924-931.

3. Drossman DA, Camilleri M, Mayer EA, Whitehead WE. AGA technical review on irritable bowel syndrome. Gastroenterology 2002;123:21082131.

4. Nellesen D, Yee K, Chawla A, Lewis BE, Carson RT. A systematic review of the economic and humanistic burden of illness in irritable bowel syndrome and chronic constipation. J Manag Care Pharm 2013;19:755764.

5. Kanazawa M, Hongo M, Fukudo S. Visceral hypersensitivity in irritable bowel syndrome. J Gastroenterol Hepatol 2011;26(suppl 3):119-121.

6. Ludidi S, Mujagic Z, Jonkers D, et al. Markers for visceral hypersensitivity in patients with irritable bowel syndrome. Neurogastroenterol Motil 2014;26:1104-1111.

7. Mertz H, Naliboff B, Munakata J, Niazi N, Mayer EA. Altered rectal perception is a biological marker of patients with irritable bowel syndrome. Gastroenterology 1995;109:40-52.

8. Patel P, Bercik P, Morgan DG, et al. Prevalence of organic disease at colonoscopy in patients with symptoms compatible with irritable bowel syndrome: cross-sectional survey. Scand J Gastroenterol 2015;50:816823.

9. Coste B, Mathur J, Schmidt M, et al. Piezo1 and Piezo2 are essential components of distinct mechanically activated cation channels. Science
2010;330:55-60.

10. Bagriantsev SN, Gracheva EO, Gallagher PG. Piezo proteins: regulators of mechanosensation and other cellular processes. J Biol Chem 2014;289:31673-31681.

11. Ferrari LF, Bogen O, Green P, Levine JD. Contribution of Piezo2 to endothelium-dependent pain. Mol Pain 2015;11:65.

12. Öhman L, Törnblom H, Simrén M. Crosstalk at the mucosal border: importance of the gut microenvironment in IBS. Nat Rev Gastroenterol Hepatol 2015;12:36-49.

13. Long Y, Liu Y, Tong J, Qian W, Hou X. Effectiveness of trimebutine maleate on modulating intestinal hypercontractility in a mouse model of postinfectious irritable bowel syndrome. Eur J Pharmacol 2010;636:159165.

14. Castro GA, Fairbairn D. Carbohydrates and lipids in Trichinella spiralis larvae and their utilization in vitro. J Parasitol 1969;55:51-58.

15. Wang H, Gong J, Wang W, et al. Are there any different effects of Bifidobacterium, Lactobacillus and Streptococcus on intestinal sensation, barrier function and intestinal immunity in PI-IBS mouse model? PLoS One 2014;9:e90153.

16. Al-Chaer ED, Kawasaki M, Pasricha PJ. A new model of chronic visceral hypersensitivity in adult rats induced by colon irritation during postnatal development. Gastroenterology 2000;119:1276-1285.

17. Xu D, Gao J, Gillilland M 3rd, et al. Rifaximin alters intestinal bacteria and prevents stress-induced gut inflammation and visceral hyperalgesia in rats. Gastroenterology 2014;146:484-496, e4.

18. Bueno L, Beaufrand C, Theodorou V, Andro-Delestrain MC. Influence of simethicone and alverine on stress-induced alterations of colonic permeability and sensitivity in rats: beneficial effect of their association. J Pharm Pharmacol 2013;65:567-573.

19. Al-Hassi HO, Bernardo D, Murugananthan AU, et al. A mechanistic role for leptin in human dendritic cell migration: differences between ileum and colon in health and Crohn's disease. Mucosal Immunol 2013;6:751-761.

20. Leser TD, Mølbak L. Better living through microbial action: the benefits of the mammalian gastrointestinal microbiota on the host. Environ Microbiol 2009;11:2194-2206.

21. Liu H, Ivarsson E, Dicksved J, Lundh T, Lindberg JE. Inclusion of chicory (Cichorium intybus L.) in pigs' diets affects the intestinal microenvironment and the gut microbiota. Appl Environ Microbiol 2012;78:4102-4109.

22. Li G, Yang M, Zhou K, et al. Diversity of duodenal and rectal microbiota in biopsy tissues and luminal contents in healthy volunteers. J Microbiol Biotechnol 2015;25:1136-1145.

23. Wang F, Knutson K, Alcaino C, et al. Mechanosensitive ion channel Piezo2 is important for enterochromaffin cell response to mechanical forces. J Physiol 2017;595:79-91.

24. Knutson K, Wang F, Alcaino C, Bernard C, Farrugia G, Beyder A. The mechanosensitive ion channel Piezo2 is selectively expressed in enterochromaffin (EC) cells of the human and mouse gastrointestinal mucosa. FASEB J 2016;30(1 suppl):1254-1255.

25. Keszthelyi D, Troost FJ, Jonkers DM, et al. Visceral hypersensitivity in 
irritable bowel syndrome: evidence for involvement of serotonin metabolism--a preliminary study. Neurogastroenterol Motil 2015;27:1127-1137. 26. O'Mahony SM, Bulmer DC, Coelho AM, et al. 5- $\mathrm{HT}_{2 \mathrm{~B}}$ receptors modulate visceral hypersensitivity in a stress-sensitive animal model of brain-gut axis dysfunction. Neurogastroenterol Motil 2010;22:573-578, e124. 